\title{
BMJ Open Pregnancy-associated cancers and birth outcomes in children: a Danish and Swedish population-based register study
}

\author{
Natalie C Momen, ${ }^{1}$ Linn Håkonsen Arendt, ${ }^{2}$ Andreas Ernst, ${ }^{2}$ Jørn Olsen, ${ }^{1,3}$ \\ Jiong Li, ${ }^{1}$ Mika Gissler, ${ }^{4,5}$ Cecilia H Ramlau-Hansen ${ }^{2}$
}

To cite: Momen NC, Arendt LH, Ernst A, et al. Pregnancyassociated cancers and birth outcomes in children: a Danish and Swedish population-based register study. BMJ Open 2018;8:e22946. doi:10.1136/ bmjopen-2018-022946

- Prepublication history and additional material for this paper are published online only. To view these files, please visit the journal online (http://dx.doi. org/10.1136/bmjopen-2018022946).

Received 14 March 2018 Revised 20 June 2018 Accepted 5 0ctober 2018

\section{Check for updates}

(c) Author(s) (or their employer(s)) 2018. Re-use permitted under CC BY-NC. No commercial re-use. See rights and permissions. Published by BMJ.

\section{${ }^{1}$ Department of Clinical}

Epidemiology, Aarhus University Hospital, Aarhus N, Denmark ${ }^{2}$ Department of Public Health, Aarhus University, Aarhus,

\section{Denmark}

${ }^{3}$ Department of Epidemiology, Fielding School of Public Health, University of California Los Angeles (UCLA), Los Angeles, California, USA

${ }^{4}$ National Institute for Health and Welfare (THL), Helsinki, Finland ${ }^{5}$ Department of Neurobiology, Karolinska Institute, Stockholm, Sweden

Correspondence to Dr Natalie C Momen; ncm@econ.au.dk

\section{ABSTRACT}

Objectives This study aims to estimate the association between pregnancy-associated maternal cancers, diagnosed both prenatally and postnatally, and birth outcomes.

Design Population-based register study.

Setting National registers of Denmark and Sweden. Participants A total of 5523365 children born in Denmark (1977-2008) and Sweden (1973-2006). Primary and secondary outcome measures: gestational age, birth weight, size for gestational age, Apgar score, caesarean section and sex were the outcomes of interest. ORs and relative risk ratios (RRR) with $95 \%$ Cls were estimated using logistic regression and multinomial logistic regression, respectively.

Results In this study, $2 \%$ of children were born to mothers with a diagnosis of cancer. Children whose mothers received a prenatal cancer diagnosis had higher risk of being born preterm (RRR: $1.77,95 \% \mathrm{Cl} 1.64$ to 1.90); low birth weight (RRR 1.84, 95\% Cl 1.69 to 2.01); low Apgar score (OR 1.36, $95 \% \mathrm{Cl} 1.20$ to 1.56); and by caesarean section (OR: $1.69,95 \% \mathrm{Cl} 1.59$ to 1.80 ). Associations moved towards the null for analyses using postnatal diagnoses, but preterm birth (RRR: $1.13,95 \% \mathrm{Cl}$ 1.09 to 1.17 ) and low birth weight (RRR: $1.14,95 \% \mathrm{Cl}$ 1.09 to 1.18 ) remained statistically significant, while risk of caesarean section became so (OR: $0.95,95 \% \mathrm{Cl} 0.91$ to 0.98). Additionally, statistical significance was reached for large for gestational age (RRR: $1.06,95 \% \mathrm{Cl} 1.01$ to 1.11), high birth weight (RRR: $1.04,95 \% \mathrm{Cl} 1.01$ to 1.06 ) and caesarean section (OR: $0.95,95 \% \mathrm{Cl} 0.91$ to 0.98 ). Conclusions Results suggest an association between pregnancy-associated cancers and adverse birth outcomes in the offspring. While this is strongest for prenatally diagnosed cancers, some smaller associations exist for postnatally diagnosed cancers, indicating that cancer itself could affect fetal development, or that cancer and adverse birth outcomes share risk factors. Future studies on maternal cancer during pregnancy should consider including some postnatal years in their exposure window.

\section{INTRODUCTION}

The fetal environment is believed to have a programming effect on child and adult health. Studies have linked adverse birth
Strengths and limitations of this study

- The study makes use of Danish and Swedish national registers to provide a large population in which to study a relatively rare exposure, pregnancy-associated cancers.

- Previous studies include maternal cancer diagnoses made up to 2 years after pregnancy, however long latency times for some cancers mean they may not capture all cancers initiated before or during pregnancy; our study is the first to extend the exposure window and investigate associations with maternal cancers diagnosed later in postnatal life.

- The potential for reverse causation and immortal observation time bias needs to be considered, although we expect their impact to be minimal.

- The study uses the entire populations of two countries, but only considers live births; if maternal cancer increases stillbirths, it would lead to an underestimation of the effects of maternal cancer in the study.

outcomes, such as low birth weight (LBW), preterm birth, small for gestational age (SGA) and low Apgar score, to neonatal morbidity and mortality, ${ }^{1-3}$ as well as later life diseases. ${ }^{4-9}$ Therefore, improving knowledge of the causes of adverse birth outcomes can aid understanding of the aetiology and possible prevention of some health conditions.

Pregnancy-associated cancers (PAC) are estimated to occur in 1 in 1000-1500 pregnancies ${ }^{10-12}$ and have been suggested to be linked to birth outcomes. ${ }^{13-20}$ Generally, PACs are defined as cancers diagnosed during pregnancy or within 1 or 2 years after childbirth. ${ }^{15-172122}$ However, long periods can pass between initial mutation of a cell until the cancer can be diagnosed and treatment initiated. ${ }^{23}$ Therefore, cancers diagnosed during the postpartum years may have been present during pregnancy but undetected, 
presenting challenges to the fetus long before a diagnosis is made.

The associations between PAC and various birth outcomes have been the subject of some research. Conclusions have been mixed, however, a number of these studies have observed increased risks of preterm birth, ${ }^{13} 15171822$ LBW, ${ }^{1418-20}$ SGA $^{1317}$ and caesarean section (CS). ${ }^{1724}$ Associations between PAC and birth outcomes may be due to cancer treatments or the stress a pregnant woman experiences when diagnosed with cancer. ${ }^{25-27}$ However, the cancer itself could play a role, through pathways such as poor nutritional status, compromised delivery of oxygen and nutrients, and chronic inflammation. ${ }^{1328-34}$

This large population-based register study aims to investigate the association between PAC and birth outcomes. We hypothesised that adverse birth outcomes would be more frequent among children of mothers diagnosed with cancer in the 'prenatal period' (2 years before pregnancy, up until childbirth) compared with children whose mothers did not receive a cancer diagnosis; due to effects of both the cancer itself and its treatment. Additionally, we expected that there would also be an increase among those born to mothers diagnosed in the 'postnatal period' (although a smaller one than for the 'prenatal period'), as a consequence of the presence of the cancer itself. However, if cancer treatment, maternal stress or lifestyle changes following diagnosis influence birth outcomes, rather than the cancer itself having an effect, any observed risk should be limited to children of mothers diagnosed in the period before the child's birth. We also included delivery by CS as an outcome, expecting that these exposed children would often be delivered early, especially to prenatally diagnosed mothers. Additionally, because research has suggested that boys are more vulnerable to in utero insults, ${ }^{35}$ we expected the male to female sex ratio to be lower among the exposed.

\section{MATERIALS AND METHODS \\ Study participants and follow-up}

All residents of Denmark and Sweden are assigned a civil registration number at birth or immigration, which can be used to accurately link data from the different nationwide registers. In this study, we identified 5523365 children born from 1 January 1977 to 31 December 2008 in Denmark and from 1 January 1973 to 31 December 2006 in Sweden, and obtained information on their birth outcomes and whether their mothers received cancer diagnoses.

\section{Ascertainment of maternal cancer diagnoses}

Maternal cancer diagnoses were extracted from the Danish Cancer Register and the Swedish Cancer Register. The registration and coding of cancers in Denmark and Sweden have been described previously. ${ }^{36} 37$

The main exposure of interest was any maternal cancers (International Classification of Diseases Seventh Revision (ICD-7) codes: 140-207 and ICD-10 codes: C00-C97) diagnosed from 2 years prior to the index child's birth, up to 15 years post partum. These data were linked to data in the Danish Civil Registration System and the Swedish Population Register on the birth dates of the child, to identify diagnoses in the exposure window. We also considered timing of the diagnosis in relation to the in utero period; prenatally (from 2 years prior to pregnancy, until the child's birth), postnatally (further divided into $<6$ years post partum; and $6-15$ years post partum) or both prenatally and postnatally (ie, those who received a cancer diagnosis both prenatally and postnatally). The exposure windows selected were arbitrary, as latency times of cancers vary and we cannot know when a cancer was initiated. Follow-up times that we believed to be reasonable were selected. Division of the exposure window was used to see if any observed increase in risk would return to normal when it became less likely that the cancer had been present during pregnancy/advanced enough during pregnancy to affect the fetus.

Furthermore, type of cancer, according to the ICD classification groups, was considered (online supplementary table 1).

\section{Ascertainment of adverse birth outcomes}

The birth outcomes included in the study were: gestational age (preterm $<37$ weeks, term $37-40$ weeks, postterm $>40$ weeks), birth weight ( LBW $<2500 \mathrm{~g}$, normal birth weight $\geq 2500-3999 \mathrm{~g}$, high birth weight (HBW) $\geq 4000 \mathrm{~g}$ ), size for gestational age (SGA, appropriate for gestational age (AGA), large for gestational age (LGA)), 5 min Apgar score (low 0-6, normal 7-10), CS (yes, no) and sex (male, female). Data were obtained from the Danish Medical Birth Registry ${ }^{38}$ and the Swedish Medical Birth Register. ${ }^{39}$ From 1994, information on CS in Denmark was obtained from the National Patient Register (ICD-10 codes O82.0O82.9 and O84.2).

Size for gestational age was calculated by ascertaining expected birth weight using sex-specific fetal growth curves for gestational age. ${ }^{40}$ A child with a birth weight $\leq 2$ SDs of expected birth weight was SGA. Those with a birth weight $>2 \mathrm{SD}$ above expected birth weight were LGA; if birth weight was between -2 SD and 2 SD of expected birth weight the child was classified as AGA.

\section{Covariates}

Each outcome of interest was considered in a crude analysis, and with adjustment for selected covariates. We identified the following available covariates using directed acyclic graphs and included them in relevant models: maternal age at time of birth (15-26 years, 27-30 years, $\geq 31$ years), child's birth year $(<1980,1980-1989,1990$ $1999, \geq 2000)$, maternal highest education at time of childbirth (low, $<9$ years, $9-14$ years, $\geq 15$ years, missing), parity $(1,2, \geq 3$, missing) and child's country of birth (Denmark, Sweden). Sex, maternal age group at time of childbirth ( $<40$ years, $\geq 40$ years) and multiplicity (singleton, twin or more) were considered to be potential effect modifiers. 


\section{Statistical analysis}

ORs with 95\% CIs were estimated for 5 min Apgar score, $\mathrm{CS}$ and sex using logistic regression. Relative risk ratios (RRR) with 95\% CIs were estimated for birth weight, gestational age and size for gestational age using multinomial logistic regression. Statistical significance was considered to have been met when the null hypothesis value of 1 was not included in the 95\% CI. Sensitivity analyses were run limiting the postpartum exposure window to 10 years, rather than 15 years, to see whether assuming shorter latency periods attenuated results. Reported analyses used complete case analysis, excluding individuals with missing data for relevant variables; however, subanalyses were run treating missing data as separate categories.

Analyses on gestational age, birth weight, size for gestational age, 5 min Apgar score and CS as outcomes were adjusted for maternal age, birth year and country. Further, in a subanalysis, adjustment was made for maternal education (as there was a higher proportion of missing values for this variable). These analyses were stratified by sex, maternal age group and multiplicity. In analyses with sex as an outcome we adjusted for maternal age and country, with further adjustment for maternal education. Stratification by maternal age group and multiplicity was also conducted. All analyses were carried out in Stata V.11, with robust estimation, allowing for some mothers having more than one child.

\section{Patient involvement}

No patients were involved in the development of the research question, the outcome measures, the design or the implementation of the study. There are no plans to disseminate the results of the research directly to study participants.

\section{RESULTS}

\section{Characteristics of the study population}

In this study of 5523365 children, 2\% ( $\mathrm{n}=95672)$ were born to a mother diagnosed with cancer from 2 years prior to the index pregnancy, to 15 years post partum ('exposed'). Of these, 9598 (10\%) were in the prenatal diagnosis group, $85674(90 \%)$ in the postnatal diagnosis group and $400(<1 \%)$ in both the prenatal and postnatal diagnosis group. Table 1 shows there were higher proportions of children born by CS, and who were LBW, preterm or SGA in the exposed group than the unexposed group. Table 1 also displays information on additional characteristics of each group. All characteristics were statistically significantly different between the exposed and unexposed groups, except sex $(\mathrm{p}<0.01)$; but in summary, exposed children were more likely to be born in Denmark, to older, multiparous mothers.

The maternal cancer type the greatest number of children were exposed to was breast cancer $(n=87077,1.6 \%)$. This was followed by female genital cancer $(n=32985$, $0.6 \%)$, skin cancer $(\mathrm{n}=21385,0.4 \%)$, digestive cancer $(\mathrm{n}=21045,0.4 \%)$ and respiratory cancer $(\mathrm{n}=11529$, $0.2 \%)$.

\section{Associations between any type of maternal cancer and adverse birth outcomes}

Figure 1 displays the associations between adverse birth outcomes and maternal cancer diagnoses for children whose mothers (1) received only prenatal diagnoses, (2) only postnatal diagnoses, and (3) diagnoses at any time point in the exposure window (all cancer diagnoses), compared with children of mothers who did not receive a cancer diagnosis.

Among children whose mothers received a prenatal diagnosis there was an increased risk of being born preterm (RRR: $1.76,95 \%$ CI 1.63 to 1.90 ) and a decreased risk of being born post-term (RRR: $0.94,95 \%$ CI 0.89 to $0.99)$. The RRR for LBW among 'prenatally exposed' was 1.83 (95\% CI 1.68 to 2.00 ), compared with the unexposed. Increases in the odds of being born by CS (OR: $1.68,95 \%$ CI 1.58 to 1.78 ) or with a low Apgar score (OR: $1.35,95 \%$ CI 1.18 to 1.53 ) were observed. SGA was elevated among the exposed (RRR: 1.12, 95\% CI 0.99 to 1.26), however the RRR for being LGA was 0.99 (95\% CI 0.88 to 1.11 ). There was no difference in sex ratio between children exposed following a prenatal maternal diagnosis and unexposed children (OR: 0.99, 95\% CI 0.95 to 1.03 ).

Associations between maternal cancer diagnosed postnatally and preterm birth (RRR: 1.13, 95\% CI 1.10 to 1.17) and LBW (RRR: 1.14, 95\% CI 1.10 to 1.18) were positive but less strong than for cancer diagnosed prenatally. There was a small increase in LGA among the exposed (RRR: $1.05,95 \%$ CI 1.01 to 1.10 ) and for HBW (RRR: $1.03,95 \%$ CI 1.01 to 1.05 ). Other results did not reach statistical significance. We observed that point estimates went towards the null in the children whose mothers were diagnosed $6-15$ years post partum compared with the group whose mothers were diagnosed $<6$ postpartum years (online supplementary figure 1).

Associations for the whole exposure window showed there was an increased risk of being preterm (RRR: $1.20,95 \%$ CI 1.17 to 1.24 ) or LBW among the exposed (RRR: $1.21,95 \%$ CI 1.17 to 1.25 ). Smaller increases were observed for HBW (RRR: 1.03, 95\% CI 1.01 to 1.05), SGA (RRR: 1.04, 95\% CI 1.00 to 1.08), LGA (RRR: $1.05,95 \%$ CI 1.01 to 1.09 ), low Apgar score (OR: $1.05,95 \%$ CI 1.00 to 1.10 ) and CS (OR: $1.08,95 \%$ CI 1.05 to 1.10 ).

Associations for the children of mothers who received cancer diagnoses in both the prenatal and postnatal periods (from 2 years prior to pregnancy up to 15 years after the child's birth) are shown in online supplementary figure 2. For these children, point estimates were most extreme, with large CIs, due to very small numbers in this exposure category.

\section{Sensitivity analyses}

The addition of socioeconomic status, in the form of maternal education level at the time of birth, to each of the models did not alter the findings; and stratified 
Table 1 Descriptive characteristics of the study population according to maternal cancer diagnosis status

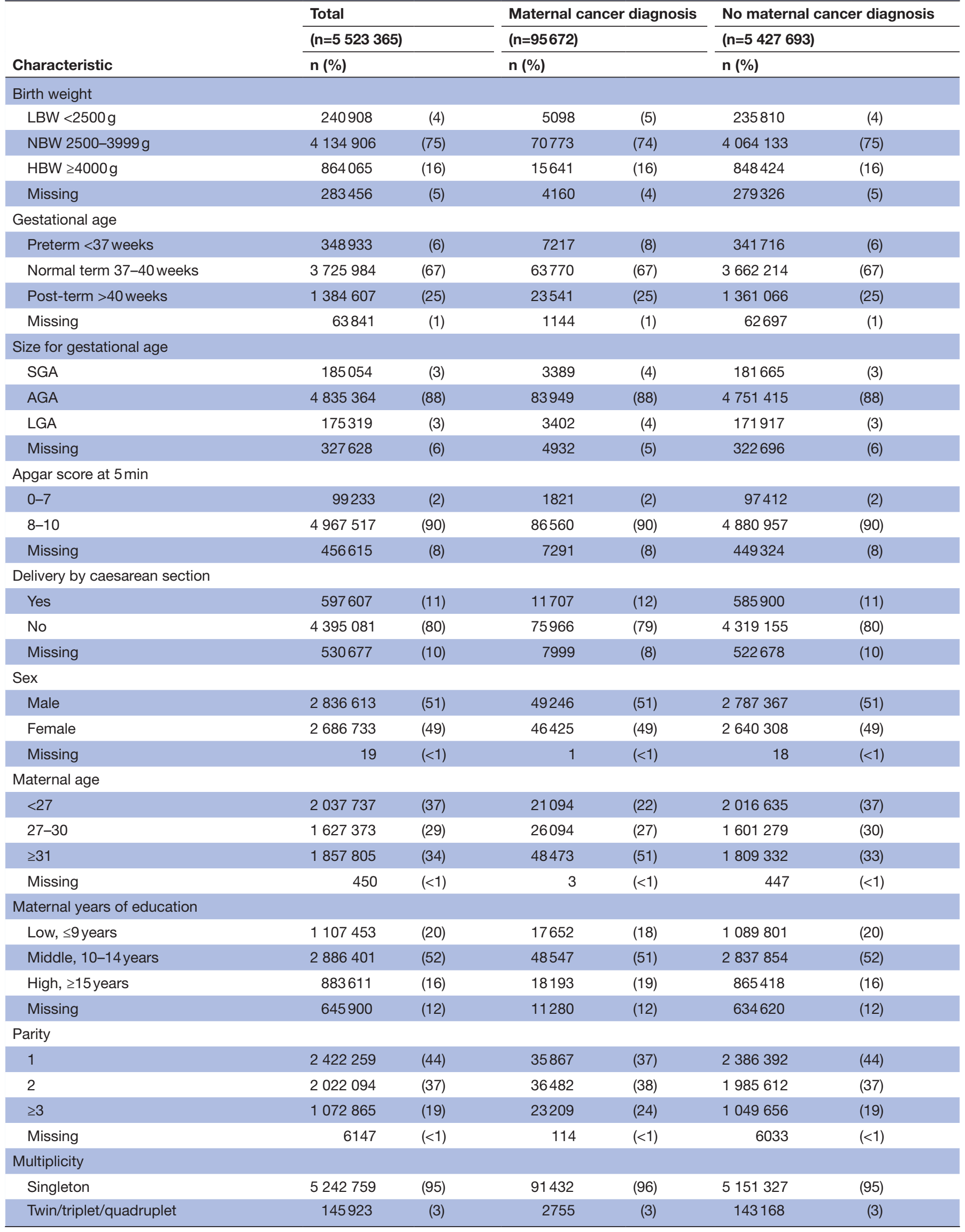


Table 1 Continued

\begin{tabular}{|c|c|c|c|c|c|c|}
\hline \multirow[b]{3}{*}{ Characteristic } & \multicolumn{2}{|l|}{ Total } & \multicolumn{2}{|c|}{ Maternal cancer diagnosis } & \multicolumn{2}{|c|}{ No maternal cancer diagnosis } \\
\hline & \multicolumn{2}{|c|}{$(n=5523365)$} & \multicolumn{2}{|l|}{$(n=95672)$} & \multicolumn{2}{|c|}{$(n=5427693)$} \\
\hline & n (\%) & & n (\%) & & n (\%) & \\
\hline \multicolumn{7}{|l|}{ Birth year group } \\
\hline$<1980$ & 897032 & $(16)$ & 15843 & $(17)$ & 881189 & $(16)$ \\
\hline$\geq 2000$ & 1270196 & (23) & 11937 & $(12)$ & 1258259 & (23) \\
\hline \multicolumn{7}{|l|}{ Country } \\
\hline Denmark & 2123229 & (38) & 37360 & (39) & 2085869 & (38) \\
\hline Sweden & 3400136 & $(62)$ & 58312 & (61) & 3341824 & (62) \\
\hline
\end{tabular}

AGA, appropriate for gestational age; HBW, high birth weight; LBW, low birth weight; LGA, large for gestational age; NBW, normal birth weight; SGA, small for gestational age.

analyses by sex did not highlight any major differences between men and women.

Limiting the postnatal exposure window to 10 years post partum resulted in 74791 exposed children (1\%). The results of the sensitivity analyses did not differ significantly from those of the main analysis.

\section{Associations between types of cancer and adverse birth outcomes}

Table 2 shows associations for birth outcomes with exposure to specific maternal cancer types. Associations reached statistical significance for almost all birth outcomes when considering maternal diagnoses of respiratory cancers (except sex) and lip, oral and pharyngeal cancers (except sex and Apgar score). Some RRRs following exposure to maternal breast cancer reached statistical significance: 1.05 (95\% CI 1.02 to 1.08$)$ for preterm birth and 0.97 (95\% CI 0.95 to 0.98 ) for post-term birth; 0.93 (95\% CI 0.89 to 0.96 ) for SGA; and 1.05 (95\% CI 1.02 to 1.07 ) for caesarean delivery. Among those born to mothers diagnosed with female genital cancers the risk for preterm birth was 1.28 (95\% CI 1.23 to 1.34); for LBW 1.32 (95\% CI 1.26 to 1.39 ); for SGA 1.15 (95\% CI 1.09 to 1.12 ) and for LGA 1.14 (95\% CI 1.07 to 1.21); for low Apgar score 1.12 (95\% CI 1.03 to 1.21); and for caesarean delivery 1.06 (95\% CI 1.02 to 1.10 ). Exposure to maternal endocrine cancer gave an RRR for preterm birth of 1.12 (95\% CI 1.04 to 1.22$)$ and for post-term birth 1.09 (95\% CI 1.04 to 1.14); for HBW 1.16 (95\% CI 1.10 to 1.22); for LGA 1.17 (95\% CI 1.05 to 1.30 ); and for caesarean delivery 1.12 (95\% CI 1.05 to 1.19$)$. An increased risk was also seen for preterm birth following exposure to maternal respiratory cancers $(1.50,95 \% \mathrm{CI} 1.40$ to 1.60$)$, and there was a reduction in RRR for post-term birth $(0.75,95 \%$ CI 0.71 to 0.79$)$. With lymphomas, risks were raised for preterm birth (1.23, 95\% CI 1.12 to 1.34$)$, LBW $(1.20,95 \%$ CI 1.08 to 1.34$)$ and HBW (1.08, 95\% CI 1.02 to 1.15$)$. Low Apgar score was observed more often among those born to mothers diagnosed with respiratory cancer (OR: 1.16,
$95 \%$ CI 1.02 to 1.33$)$ or lymphoma $(1.19,95 \%$ CI 1.01 to 1.41). Sex ratio was not different between the exposed and unexposed for any specific type of cancer, whereas CS was more frequent following exposure to any cancer type.

When considering type of cancer by timing of diagnosis (online supplementary table 2), numbers became small, particularly for prenatal diagnoses. However, statistical significance was reached for digestive cancers and caesarean delivery; mesothelial and soft tissue cancer and low Apgar score; breast cancers with preterm birth, LBW and caesarean delivery; female genital cancers with preterm birth, LBW, HBW and caesarean delivery; and CNS cancers and caesarean delivery. Results for postnatal diagnoses of specific groups of cancer were very similar to those seen for cancers diagnosed across the whole exposure window.

\section{DISCUSSION \\ Main findings}

Prenatal diagnosis of a maternal cancer from the 2 years before pregnancy up to childbirth was associated with preterm birth, post-term birth, LBW, SGA, low Apgar score and CS. Associations were smaller for postnatal diagnosis remained elevated for preterm birth and LBW. LGA was also higher among those postnatally exposed; whereas CS was negatively associated with postnatal maternal cancer diagnoses.

\section{Interpretation}

Adverse birth outcomes indicate a suboptimal fetal environment and have been observed to be associated with certain factors which could reasonably be expected to exist when a pregnant woman has cancer: poor nutritional intake, compromised placental function resulting in poor delivery of oxygen and nutrients to the fetus, and chronic inflammation. ${ }^{13}{ }^{28-34}$ Yet, the associations seen between maternal cancers diagnosed prenatally and adverse birth outcomes could also be due to cancer 
Birth outcomes

i) Prenatal exposure only

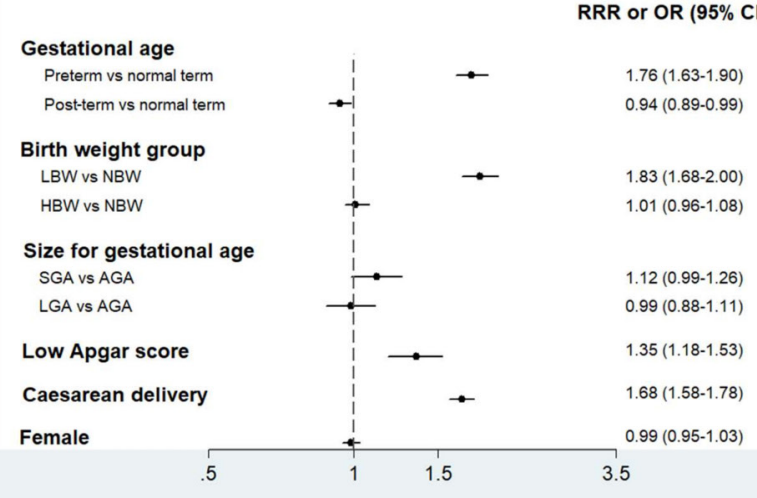

ii) Postnatal exposure only

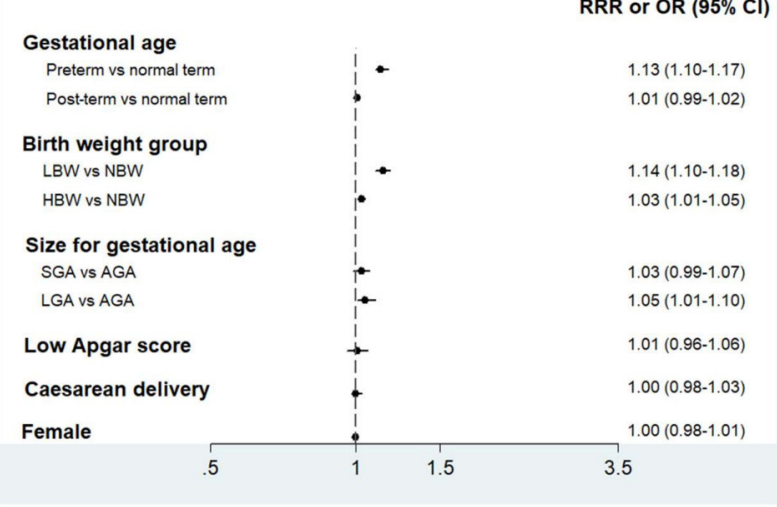

iii) All exposed (maternal diagnosis at any timepoint in exposure window

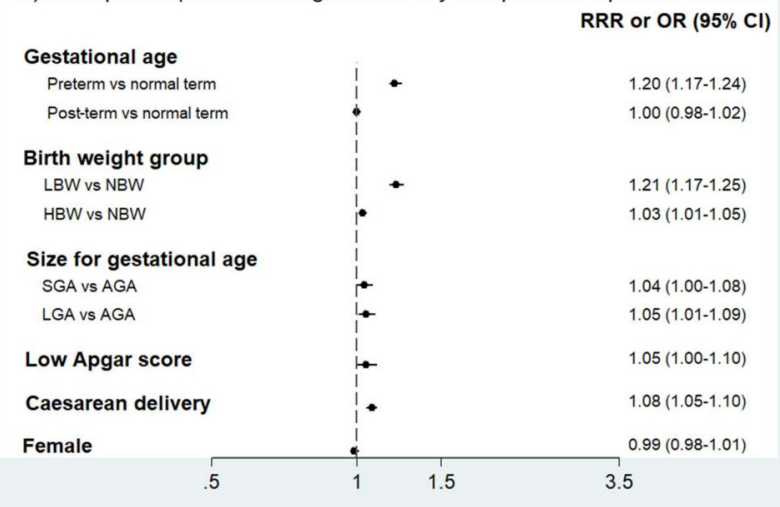

Figure 1 Associations for birth outcomes according to maternal cancer diagnosis status among children born in Denmark (1977-2008) and Sweden (1973-2006). AGA, appropriate for gestational age; HBW, high birth weight; LBW, low birth weight; LGA, large for gestational age; NBW, normal birth weight; RRR, relative risk ratio; SGA, small for gestational age.

treatments, stress or lifestyle changes following a diagnosis, rather than the effects of the cancer itself. ${ }^{25} 2741$ CS could be the preferred mode of delivery ${ }^{42}{ }^{43}$ and early delivery by CS or induction may be preferable to allow treatments to commence. These medical interventions to deliver a child early due to a maternal cancer would cause iatrogenic LBW or preterm birth. It is probable that the larger risks seen among the prenatally exposed for LBW and preterm birth in our study are driven by the increased frequency of CS also observed in this group. However, higher SGA was also observed, which would be a stronger indicator of intrauterine growth restriction. Alternatively, there may be common causes for birth outcomes and cancer, with something the mother was exposed to earlier in life influencing her risk of cancer and her offspring's risk of adverse birth outcomes.

Findings from previous studies on the association between PAC and birth outcomes have focused on prenatal diagnoses and up to 2 years after childbirth, and have been equivocal. The results from analyses on prenatal cancer diagnosis exposure are in line with some of these findings; for example, increased risk of preterm birth ${ }^{17} 1842$ with any type of cancer, or with breast cancer or Hodgkin's disease during pregnancy. ${ }^{15} 22$ LBW has also been seen to be higher among children of mothers with cancer in a number of studies. ${ }^{14-20}$ However, other studies have reported no increase in risk of preterm birth $^{161944}$ or LBW. ${ }^{15162244}$ Our study supports findings of increases in risk of SGA have been seen among children exposed to any cancer ${ }^{174}$ or ovarian cancers ${ }^{17}$; however, we did not find increases in risk following exposure to haemopoetic cancers as seen in a study. ${ }^{17}$ Apgar score has been studied in offspring of mothers who received cancer treatment and was not found to be lower among exposed children. ${ }^{135}$ While we found an increase in low Apgar score among those exposed to any cancer and some specific types of cancer, we were not able to also look at cancer treatments. Sex ratio has been looked at in two studies, both of which found a higher ratio of male to female liveborns ${ }^{15}{ }^{16}$; however, we did not see a difference between the unexposed and exposed. CS has been found to be higher among children of mothers diagnosed prenatally with any cancer ${ }^{17}$ and postnatally with breast cancer. ${ }^{24}$ In summary, our study supports some of these previous findings, in a large, multinational study population, and suggests that the postnatal period considered in some papers could potentially be extended.

Associations seen for children of women diagnosed in the $6-15$ years postpartum period suggested more normal perinatal health indicators than for those of women diagnosed $<6$ years post partum. However, while this could indicate that the cancer process had not started yet, it could also mean that if the disease process was ongoing, it had not reached a stage that interfered with fetal growth and development.

\section{Strengths and limitations}

The main strength of this study is the use of nationwide data from Swedish and Danish register systems. These cover the two countries' entire populations, with obligatory data collection and minimal selection bias. Data from the cancer registries and birth registers are considered to be of high quality and completeness. ${ }^{36-39}$ However, even with these large data sets, it is difficult to look at associations with specific types of cancer because they are not common in these age groups. 


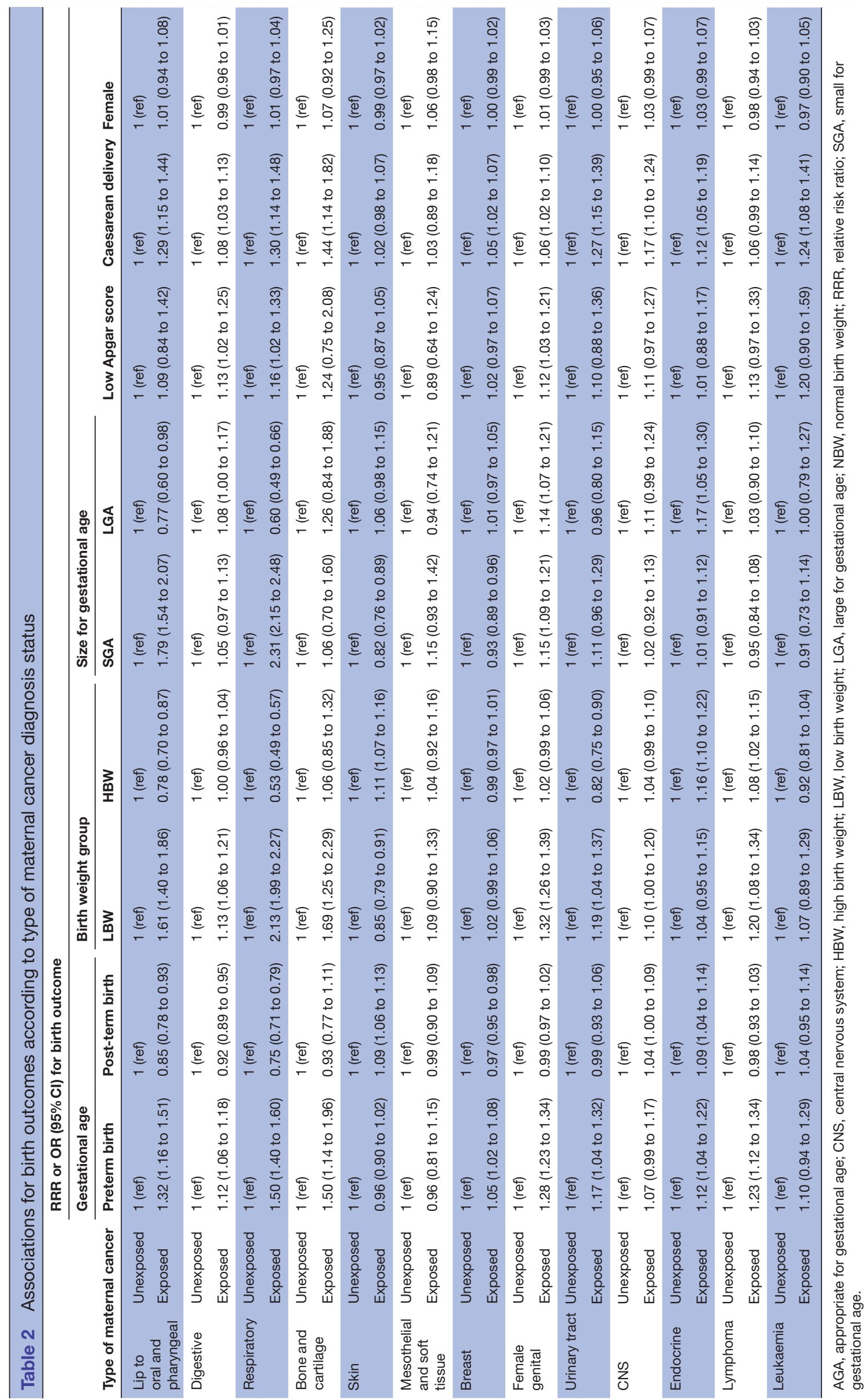


The study considers PAC in a novel way. Previous studies have limited their definition to a maximum of 2 years post partum. However, the minimum latency periods to clinical diagnoses for cancers in adults were determined by the World Trade Center Health Program to be 0.4 years for lymphoproliferative and haematopoietic cancers and 4 years for solid tumours (excluding mesotheliomas (11 years) and thyroid cancers (2.5 years)), with the induction and latency times of some cancers being much longer. ${ }^{23}$ This suggests that some cancers present during pregnancy would not have been included in previous research. Limiting the exposure window to 15 years post partum was an arbitrary decision. Some cancers diagnosed towards the end of the window may not have been present during pregnancy, or cancers diagnosed after this point were present but undetected. However, reducing the exposure window to 10 years post partum did not alter results. Future research could consider the postpartum period further and investigate at what time point after birth maternal cancers were no longer associated with adverse birth outcomes.

Although selection bias is minimised by inclusion of the entire populations of Denmark and Sweden, this study only considers live births. While some studies have not found an increase in infant and neonatal deaths among those exposed to maternal cancer, ${ }^{18}{ }^{2044}$ others have seen an increase in stillbirth. ${ }^{1617} 19$ This would potentially lead to an underestimation of the effects of maternal cancer in this study. ${ }^{46}$

Children in the study were considered exposed if their mother was diagnosed with cancer from 2 years before conception, until 15 years post partum. However, as not all mothers could be followed for 15 years post partum, some children will have been misclassified as unexposed.

The potential impacts of immortal observation time bias and reverse causation also need to be considered. Although a cancer may have already been initiated in the mother before a child's birth, the exposure status of the child is based on the actual diagnosis of a maternal cancer; an event which, in some cases, occurred after birth outcomes were observed. However, it is unlikely cancer would be differentially diagnosed among mothers with a preterm or LBW child. It should be considered though, for a maternal cancer diagnosis to be made and a child considered 'exposed', mothers need to survive until they receive a cancer diagnosis, not dying of alternative causes before this.

Residual confounding resulting from unmeasured factors cannot be ruled out. There are many potential confounders that we have not included in our study because they were not available throughout the study period in both countries. Some potential confounders which we were not able to control for include maternal smoking during pregnancy, ${ }^{47}$ or maternal nutrition during pregnancy. ${ }^{28}$ Furthermore, we did not have access to any information on treatment during pregnancy. Based on the small numbers of women who received a prenatal diagnosis, the numbers in any treatment groups would be small and a study looking into the potential effects of types of treatment would likely be underpowered. However, this is of interest and should be considered further in future research, either combining data from multiple Nordic registers, or in a different setting altogether. Additionally, we carried out a large number of analyses and the role of chance cannot be excluded in the observations that were considered to be statistically significant.

\section{CONCLUSION}

Our findings suggest that in utero exposure to maternal cancer is associated with adverse birth outcomes. While the association was stronger among those exposed to prenatally diagnosed cancers, the associations seen for postnatally diagnosed cancers suggest that either birth outcomes and cancer share common causes, or that the cancer itself (rather than just consequences of a diagnosis) may influence risk of adverse birth outcomes. These associations are not strong enough to justify screening for maternal cancer in mothers of children with birth complications.

Contributors The study was conceived by LHA, AE and CHRH. It was planned by LHA, AE, CHRH, NCM, JO and JL. The data analysis was performed by NCM. The manuscript was written by NCM, with revisions and final approval from LHA, AE, $\mathrm{CHRH}, \mathrm{NCM}, \mathrm{JO}, \mathrm{MG}$ and $\mathrm{JL}$.

Funding This work was supported by grants from Novo NordiskFoundation (12535), Nordic Cancer Union (176673, 186200), European ResearchCouncil (ERC-2010-StG-260242-PROGEURO), the Danish Council for IndependentResearch (DFF -6110-00019), Karen Elise Jensens Fond (2016) and Program for Clinical Research Infrastructure(PROCRIN) established by the Lundbeck Foundation and the Novo NordiskFoundation.

Competing interests None declared.

Patient consent Not required.

Ethics approval Danish Data Protection Agency (j nr 2008-41-2680), Scientific Ethics Committee of Central Jutland Region (VEK, sagnr M-20100252) and the Research Ethics Committee (EPN) at the Karolinska Institute (reference number 2008/4:6).

Provenance and peer review Not commissioned; externally peer reviewed. Data sharing statement № additional data are available.

Open access This is an open access article distributed in accordance with the Creative Commons Attribution Non Commercial (CC BY-NC 4.0) license, which permits others to distribute, remix, adapt, build upon this work non-commercially, and license their derivative works on different terms, provided the original work is properly cited, appropriate credit is given, any changes made indicated, and the use is non-commercial. See: http://creativecommons.org/licenses/by-nc/4.0/.

\section{REFERENCES}

1. Tenovuo A, Kero P, Piekkala P, et al. Fetal and neonatal mortality of small-for-gestational age infants. A 15-year study of 381 cases. Eur J Pediatr 1988;147:613-5.

2. McCormick MC. The contribution of low birth weight to infant mortality and childhood morbidity. N Engl J Med 1985;312:82-90.

3. Callaghan WM, MacDorman MF, Rasmussen SA, et al. The contribution of preterm birth to infant mortality rates in the United States. Pediatrics 2006;118:1566-73.

4. Sonnenschein-van der Voort AM, Arends LR, de Jongste JC, et al. Preterm birth, infant weight gain, and childhood asthma risk: a meta-analysis of 147,000 European children. J Allergy Clin Immunol 2014;133:1317-29.

5. Eriksson JG. Early growth, and coronary heart disease and type 2 diabetes: experiences from the Helsinki Birth Cohort Studies. Int J Obes 2006;30:S18-22. 
6. Barker DJ. The developmental origins of adult disease. J Am Coll Nutr 2004;23:588S-95.

7. Barker DJ. Fetal origins of coronary heart disease. $\mathrm{Br} \mathrm{Heart} J$ 1993;69:195-6.

8. Bhattacharya S, Beasley M, Pang D, et al. Maternal and perinatal risk factors for childhood cancer: record linkage study. BMJ Open 2014;4:e003656.

9. Li J, Cnattingus S, Gissler M, et al. The 5-minute Apgar score as a predictor of childhood cancer: a population-based cohort study in five million children. BMJ Open 2012;2:e001095

10. Pavlidis NA. Coexistence of pregnancy and malignancy. Oncologist 2002;7:573-87.

11. Lambe M, Ekbom A. Cancers coinciding with childbearing: delayed diagnosis during pregnancy? BMJ 1995;311:1607-8.

12. Lee YY, Roberts CL, Dobbins $T$, et al. Incidence and outcomes of pregnancy-associated cancer in Australia, 1994-2008: a populationbased linkage study. BJOG 2012;119:1572-82.

13. Amant $F$, Vandenbroucke $T$, Verheecke $M$, et al. Pediatric outcome after maternal cancer diagnosed during pregnancy. N Engl J Med 2015;373:1824-34.

14. Janov AJ, Anderson J, Cella DF, et al. Pregnancy outcome in survivors of advanced Hodgkin disease. Cancer 1992;70:688-92.

15. Langagergaard V, Horvath-Puho E, Nørgaard M, et al. Hodgkin's disease and birth outcome: a Danish nationwide cohort study. Br J Cancer 2008;98:183-8.

16. Langagergaard V, Puho EH, Lash TL, et al. Birth outcome in Danish women with cutaneous malignant melanoma. Melanoma Res 2007:17:31-6.

17. Lu D, Ludvigsson JF, Smedby KE, et al. Maternal cancer during pregnancy and risks of stillbirth and infant mortality. J Clin Oncol 2017:35:1522-9.

18. Smith LH, Dalrymple JL, Leiserowitz GS, et al. Obstetrical deliveries associated with maternal malignancy in California, 1992 through 1997. Am J Obstet Gynecol 2001;184:1504-13. discussion 12-3.

19. Zemlickis D, Lishner M, Degendorfer $P$, et al. Maternal and fetal outcome after breast cancer in pregnancy. Am J Obstet Gynecol 1992;166:781-7.

20. Zemlickis D, Lishner M, Degendorfer P, et al. Maternal and fetal outcome after invasive cervical cancer in pregnancy. J Clin Oncol 1991;9:1956-61.

21. Langagergaard V. Birth outcome in women with breast cancer, cutaneous malignant melanoma, or Hodgkin's disease: a review. Clin Epidemiol 2010;3:7-19.

22. Langagergaard V, Gislum M, Skriver MV, et al. Birth outcome in women with breast cancer. Br J Cancer 2006;94:142-6.

23. Howard J. Minimum Latency \& Types or Categories of Cancer. World Trade Center Health Program. 2013.

24. Kang EJ, Seo JH, Kim LY, et al. Pregnancy-Associated risk factors of postpartum breast cancer in Korea: a Nationwide Health Insurance Database Study. PLoS One 2016;11:e0168469.

25. Dole N, Savitz DA, Hertz-Picciotto I, et al. Maternal stress and preterm birth. Am J Epidemiol 2003;157:14-24.

26. Li J, Vestergaard M, Obel C, et al. A nationwide study on the risk of autism after prenatal stress exposure to maternal bereavement. Pediatrics 2009;123:1102-7.
27. Van Calsteren K, Heyns L, De Smet F, et al. Cancer during pregnancy: an analysis of 215 patients emphasizing the obstetrical and the neonatal outcomes. J Clin Oncol 2010;28:683-9.

28. Abu-Saad K, Fraser D. Maternal nutrition and birth outcomes. Epidemiol Rev 2010;32:5-25.

29. Cotechini T, Graham $\mathrm{CH}$. Aberrant maternal inflammation as a cause of pregnancy complications: A potential therapeutic target? Placenta 2015;36:960-6.

30. Brenner B, Avivi I, Lishner M. Haematological cancers in pregnancy. Lancet 2012;379:580-7.

31. Doyle W, Crawford M, Costeloe K. Maternal nutrition and birth weight. These factors are related. BMJ 2000;320:941-2.

32. Sankaran S, Kyle PM. Aetiology and pathogenesis of IUGR. Best Pract Res Clin Obstet Gynaecol 2009;23:765-77.

33. Su Q, Zhang $\mathrm{H}$, Zhang $\mathrm{Y}$, et al. Maternal stress in gestation: birth outcomes and stress-related hormone response of the neonates. Pediatr Neonatol 2015;56:376-81.

34. Toledo MT, Gomes Marcondes MC. Placental glycogen metabolism changes during walker tumour growth. Placenta 2004;25:456-62.

35. Eriksson JG, Kajantie E, Osmond C, et al. Boys live dangerously in the womb. Am J Hum Biol 2010;22:330-5.

36. Barlow L, Westergren K, Holmberg L, et al. The completeness of the Swedish Cancer Register: a sample survey for year 1998. Acta Oncol 2009;48:27-33.

37. Gjerstorff ML. The Danish Cancer Registry. Scand J Public Health 2011;39:42-5.

38. Knudsen LB, Olsen J. The Danish Medical birth registry. Dan Med Bull 1998;45:320-3.

39. Odlind V, Haglund B, Pakkanen M, et al. Deliveries, mothers and newborn infants in Sweden, 1973-2000. Trends in obstetrics as reported to the Swedish Medical Birth Register. Acta Obstet Gynecol Scand 2003;82:516-28.

40. Marsál K, Persson PH, Larsen T, et al. Intrauterine growth curves based on ultrasonically estimated foetal weights. Acta Paediatr 1996;85:843-8.

41. Li J, Johansen C, Hansen D, et al. Cancer incidence in parents who lost a child: a nationwide study in Denmark. Cancer 2002;95:2237-42.

42. Amant F, Coosemans A, Debiec-Rychter M, et al. Clinical management of uterine sarcomas. Lancet Oncol 2009;10:1188-98.

43. Zhang X, Gao YL, Yang Y. Treatment and prognosis of cervical cancer associated with pregnancy: analysis of 20 cases from a Chinese tumor institution. J Zhejiang Univ Sci B 2015;16:388-94.

44. Lishner M, Zemlickis D, Degendorfer $\mathrm{P}$, et al. Maternal and foetal outcome following Hodgkin's disease in pregnancy. $\mathrm{Br} \mathrm{J}$ Cancer 1992;65:114-7.

45. Weisz B, Schiff E, Lishner M. Cancer in pregnancy: maternal and fetal implications. Hum Reprod Update 2001;7:384-93.

46. Liew Z, Olsen J, Cui X, et al. Bias from conditioning on live birth in pregnancy cohorts: an illustration based on neurodevelopment in children after prenatal exposure to organic pollutants. Int $\mathrm{J}$ Epidemiol 2015;44:345-54.

47. Butler NR, Goldstein H, Ross EM. Cigarette smoking in pregnancy: its influence on birth weight and perinatal mortality. Br Med $\mathrm{J}$ 1972;2:127-30 